\title{
A life course approach: research orientations and future challenges
}

\author{
Eino Heikkinen
}

Received: 5 August 2010/Accepted: 20 August 2010/Published online: 11 September 2010

(C) European Group for Research into Elderly and Physical Activity (EGREPA) 2010

\begin{abstract}
The need to study lifelong changes in health and functioning has resulted in increasing emphasis on a life course approach in different fields of inquiry. The aim of this approach is to explore how biological, psychological, and social risk factor trajectories, acting across the entire life course, influence age-related diseases, functional decline, and disability. The importance of the first years of life upon later development and adult characteristics was generally recognized already in the first half of the twentieth century, but it was not until the 1990s that different strands of medical and social research converge in the field of life course epidemiology, in which epidemiological processes are approached using different models such as biological programming, critical periods, pathways, and accumulation. The biological programming model holds that organ development in utero and early infancy determines the maximum functional capacity that an individual can attain and influences the development of certain chronic diseases later in life. The critical period model extends the idea to include child development and key social transitions over the entire life course. The pathway model focuses on the cumulative effect of life events along the developmental trajectories, with early advantage or disadvantage setting a person on a pathway to a later etiologically important exposure. The accumulation model describes the underlying social, behavioral, and biological processes that drive the impact of the life course on health. The growing focus on life course determinants of aging also has implications for studies of long-term changes in physical activity and their role in determining both gains
\end{abstract}

E. Heikkinen $(\bowtie)$

Gerontology Research Centre, University of Jyväskylä,

P.O. Box 35 (VIV), 40014 Jyväskylä, Finland

e-mail: eino.heikkinen@sport.jyu.fi and losses of health and functioning with aging. A life course approach presents great challenges for the continued development of testable theoretical models and effective study design and analysis.

Keywords Life course approach · Aging · Health . Functional capability

\section{Introduction}

Much of the previous research into aging and aging-related functional decline and chronic diseases, and in consequence preventive factors such as physical activity has been conducted on middle-aged and older people. Despite increasing research efforts, it has been difficult to disentangle the human aging process, define the mechanisms and determinants of healthy aging, and identify interventions to promote healthy life expectancy of the population. Current aging research highlights the consensus that the aging process has its beginnings in early life, and that adult function and age-related chronic diseases have their origins in early life experience and share common risk factors [1]. Not only is a more holistic, interdisciplinary life course approach required but it is also fundamental that the scientific findings are translated without delay into clear messages that address the social and economic implications of an aging population [2].

This minireview outlines the models and methods of the life course approach and studies and how they have contributed to our understanding of age-related changes in function and chronic diseases. The review is divided into three sections. The first describes what a life course approach is. The second section focuses on the development of life course epidemiology. The last section deals 
with challenges of the life course approach and suggests perspectives for future research.

\section{What is a life course approach?}

Over the last few decades, growing interest has been shown in studying age-related changes in functioning and conceptualizing disease etiology within a life course framework $[1,3]$. The aim is to explore how biological, psychological, and social risk factor trajectories, acting across the whole life course, influence age-related diseases, functional decline, and disability. There is growing evidence from life course studies that adult function and age-related chronic diseases have their origins in early life experiences and share common risk actors $[1,4]$. The interest in this approach is not, however, new. The importance of the first years of life upon later development and adult characteristics, particularly with respect to biological changes, was generally recognized already in the first half of the twentieth century $[3,5]$. A more recent orientation has been the adoption of the life course perspective in human biology, where the focus is on the ways in which early environmental factors can influence human form and function across the life span [3].

In the latter part of the twentieth century, a life span perspective in developmental psychology has emerged in which psychological development is seen as a lifelong process [6]. In this perspective, individual life courses are investigated in their biocultural and sociohistorical context. From the social science point of view, a life course approach concentrates on age-related transitions that are socially created, socially recognized, and shared [7]. Sociologists have investigated how the individual's life course is structured by institutions and culture, social and historical change, and changes in individual aging processes.

According to Blane et al. [8], during the 1990s, three strands of social and medical research-biological programming, accumulation, and health inequalities - converged in life course epidemiology. The idea of biological programming holds that the development of organs and metabolism in utero and during early infancy determines the maximum functional capacity that an individual can attain and sets the limits of functional capacity for the entire life course $[8,9]$. The accumulation model implies that childhood disadvantage tends to be followed by health-relevant disadvantage across adulthood and into early old age [10]. In the study of social inequalities in health, evidence is presented on the range of social factors that are found to be associated with different aspects of health in early life, childhood, adolescence, and adulthood [11]. Uncertainty, however, exists about the continuation of this inequality into older age [12]. It is clear that among the very old, the socioeconomic factors, at least with regard to mortality, have decreased their importance [13].

\section{Life course epidemiology}

The definitions of life course epidemiology emphasize collaboration between the social and natural sciences. "The natural science process of aetiology supplies the disease outcomes, while the social science process of accumulation ensures the social patterning, by social class and such likes, of these diseases" [8].

Kuh and Ben-Shlomo [14] have defined a life course approach to chronic disease epidemiology "as the study of long-term effects on chronic disease risk of physical and social exposures during gestation, childhood, adolescence, young adulthood and late adult life. It includes studies of the biological, behavioural and psychosocial pathways that operate across an individual's life course, as well as across generations, to influence the development of chronic diseases".

The most commonly used approaches in studying life course processes are defined in terms of three, partly overlapping models: critical periods, pathways, and accumulation $[8,15]$.

The critical period model According to Ben-Shlomo and Kuh [3], "the 'critical period model' is when an exposure acting during a specific period has lasting or lifelong effects on the structure or function of organs, tissues and body systems which are not modified in any dramatic way by later experience". There is evidence suggesting, for example, that intrauterine growth retardation (as evidenced by small size at birth) may lead to a variety of chronic disorders in adult life, such as hypertension, cardiovascular disease, and non-insulin-dependent diabetes [4, 9]. This model is also known as biological programming $[8,9]$.

Exposures to risk factors in later life may nevertheless influence disease risk as modifiers. For example, the relationship of the above-mentioned chronic disorders with low birth weight has been observed in particular in subjects who become obese in later phases of life [9]. There is also evidence to suggest interaction between small size at birth and accelerated growth in height raises the risk of CHD and or metabolic syndrome [16, 17].

In adolescence, there are also critical issues that impact the development of chronic disorders in later life. A few long-term studies have shown the relationships between high blood pressure in adolescence or young adulthood and later risk of stroke or CDH in midlife [4]. This trend seems to be visible, particularly in developing countries [18]. Risk factors tend to cluster already in childhood and adolescence and associate later with cardiovascular disease [e.g., 19]. It has been observed, for instance, that the majority of overweight children have at least one additional risk factor for cardiovascular disease including raised blood pressure and dyslipidemia [20]. 
Physical activity patterns have also changed. A considerable part of the day is spent sitting at school or in front of a computer or a TV set. Increase in the consumption of sugar-rich beverages and energy-rich meals (often as "fast food" and snacks) have also occurred in many countries, increasing the risk for obesity [20]. It is obvious that healthrelevant lifestyles do not just simply develop in adulthood, but have their origins in earlier phases of life and tend to stay over the entire life course.

The critical period model has also been defined as key social transitions. From this point of view, the life course is seen as combining biological and social elements which interact with each other and produce social variation in health [21]. A life course approach might help in understanding the ways in which social factors affect the transition through "socially critical periods" [21]. Such periods include the transition from primary to secondary school, school examinations, the transition from school to work, leaving the parental home, establishing one's own residence, the transition to parenthood, job insecurity, change or loss, the onset of chronic illness, and exit from the labor market into retirement. Life course research is showing us how health at older ages has developed in different cohorts and their different subcohorts as they move through time and encounter socially critical periods characterized by specific social policy measures.

The pathway model According to the pathway model, early advantage or disadvantage in relation to etiological exposure sets a person on a pathway to a later exposure that is the etiologically important event [3, 8] Ben-Shlomo and Kuh [3] illustrate this by describing four different pathways with respect to adult respiratory disease and/or impaired respiratory function (these pathways can also be applied in the case of other continuous physiological measures such as muscle strength and speed of behavior): (1) a predominantly biological pathway exists where, for example, impaired fetal development of the lung is associated with future respiratory problems in adulthood; (2) a predominantly social pathway exists where an adverse socioeconomic position in childhood influences adverse childhood exposures as well as adult socioeconomic position and, for example, smoking behavior; (3) in the third, sociobiological, pathway, an adverse socioeconomic position in childhood is associated with post-natal lung function and subsequently with poor adult lung function though its effects on immune function and the likelihood of exposure to infectious agents are unlikely; and (4) in the fourth, the biosocial, pathway, repeated childhood infections result in adverse educational attainment and lower socioeconomic status.

The life course approach requires integration of biological and psychosocial pathways along with recognition of the fact that the timing of exposures may affect disease risk in many different ways.
The accumulation model The accumulation model suggests that factors that elevate disease risk or enhance good health may accumulate gradually over the life course. The model builds on the tendency, caused by the social structure, for advantages or disadvantages to cluster cross- sectionally and accumulate longitudinally [8]. One person may work in a hazard-free environment, reside in good quality housing in an area with little air pollution, and have an income sufficient to permit a healthy diet. In contrast, another person may have a hazardous working environment, live in poor housing conditions, and have an income which restricts dietary choice. Longitudinally, risk factors at different life stages may also accumulate over time, one adverse exposure or experience leading to another. A child raised in a poor home, for example, is more likely to fail educationally, leave school at the minimum age, enter the unskilled labor market doing hazardous work with low pay, and end up in old age in a situation of financial insecurity [22].

Figure 1 illustrates the accumulation model of the life course approach, showing the social and behavioral processes that underlie the impacts of life course on health [23].

This model has been shown to have good predictive power as, for example, in the results of the 1946 British birth cohort study [24, 25], and in several studies carried out in the Nordic countries [e.g., 26-28]. In particular, lifetime manual work is associated with various health problems, chronic disorders, physical disability, poor self-rated health, and mortality, implying that a person's level of health tends to be a function of the proportion of their life course exposed to disadvantage [8]. In consequence, a person moving upwards may be healthier than another person remaining in their class of origin. Upwardly, mobile individuals tend, however, to be less healthy than those already belonging to the higher classes, as do those moving downwards [8].

A life course approach applying the accumulation model can provide knowledge about the etiology of various health

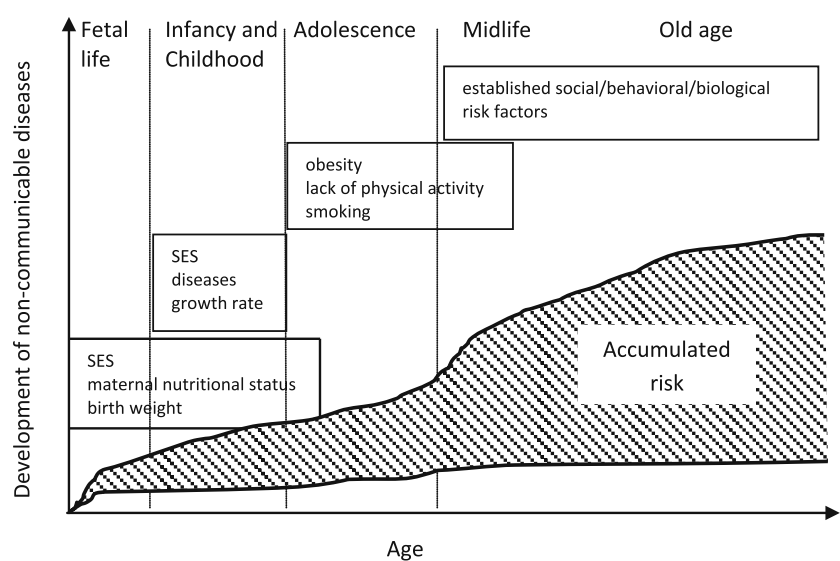

Fig. 1 The accumulation model suggests that factors that elevate disease risk or enhance good health may accumulate gradually over the life course. Adapted from Darnton-Hill et al. [4] and Aboderin et al. [23] 
problems. Some diseases such as lung cancer may derive mostly from adult circumstances, coronary heart disease, and lung disease from both childhood and adult circumstances, while stroke and stomach cancer appear to be associated with adverse social circumstances during childhood [29].

It has also been observed that adult cardiovascular risk factors relate in different ways to the life course [25]. The behavioral risk factors (tobacco smoking and recreational physical exercise) were associated primarily with adult socioeconomic circumstances, while the physiological risk factors (serum cholesterol, blood pressure, BMI, and lung function) were associated to varying extents with socioeconomic circumstances in both childhood and adulthood. These elaborations have led to the generalization that life course influences are strongest where they can be defined physiologically, i.e., in the form of health and disease, intermediate in relation to shaping preferences and taste, and are smallest in their effect on psychological reactions in everyday life [8]. There also is growing evidence suggesting that the various risk factors and their associations with socioeconomic circumstances as well as their interactions in the causation of chronic disorders and mortality are partly different in old age as compared to early old age [13]

This question is related to the hypothesis known as "reverse epidemiology", according to which some cardiovascular risk factors, such as obesity, dyslipidemia, and hypertension are not harmful but instead seem to contribute to better survival in the case of some chronic diseases or in individuals with advanced age [30]. The mechanisms of these inverse associations are not clear. Several possible causes have been hypothesized: subclinical diseases and old-age frailty [31] may modify certain risk factors (decrease in hepatic and intestinal function reduces the level of serum cholesterol, heart failure lowers blood pressure, frailty or Alzheimer's disease reduces weight, etc.), while the time discrepancy between the competing risk factors may also play a role [30]. A recent study focusing on the "obesity paradox" [32] showed that in old age, both normal weight and overweight men are a mixture of individuals whose weight trajectories have been different during their life course, but the worst prognosis in late life was found among men with overweight and high CVD risk in midlife with subsequent weight decrease.

\section{Future challenges}

The evidence presented demonstrates that the risks for disease, physical disability, and mortality are influenced by factors at all stages of the life course, and that life course influences may be specific to different health outcomes. Furthermore, risk factors, influences, and outcomes seem to show significant overlap, while socioeconomic status seems to play a major role throughout the life course; however, the associations are population-specific reflecting social, economic, cultural, ethnic, and behavioral differences.

The life course approach, applying the accumulation model, has mostly focused on the association of chronic disorders with socioeconomic circumstances in different phases of life. Currently, increasing interest is being shown in age-related changes in functional capability, using tests of physical and cognitive performance, and sensory function [1]. The reverse epidemiology with respect to the conventional risk factors for a poor clinical outcome and premature mortality deserves further investigation from the life course perspective, as it may have a bearing on the management of people at advanced age.

The lifetime development of different capabilities shows a rapid rise in early years, peaking on average between 20 and 30 years of age and then either maintaining a plateau over a couple of decades or starting a gradual decline at about 30 years of age [33]. There are wide inter-individual differences in the peak level of capability attained and in the age at which the decline exceeds the critical threshold of functional limitations. There are discontinuities in both the attainment of the peak level and in the decline with aging. It is not yet known to what extent the levels of physical and cognitive capability and their rates of changes are associated with each other, and under what circumstances and at what ages these capabilities begin to differentiate, and what are the risk factors for this differentiation [1]. The paradox of reverse epidemiology regarding the risks of chronic disorders and mortality in old age [30,32] and frailty as an emerging geriatric syndrome [31] also deserve further investigations applying the life course approach.

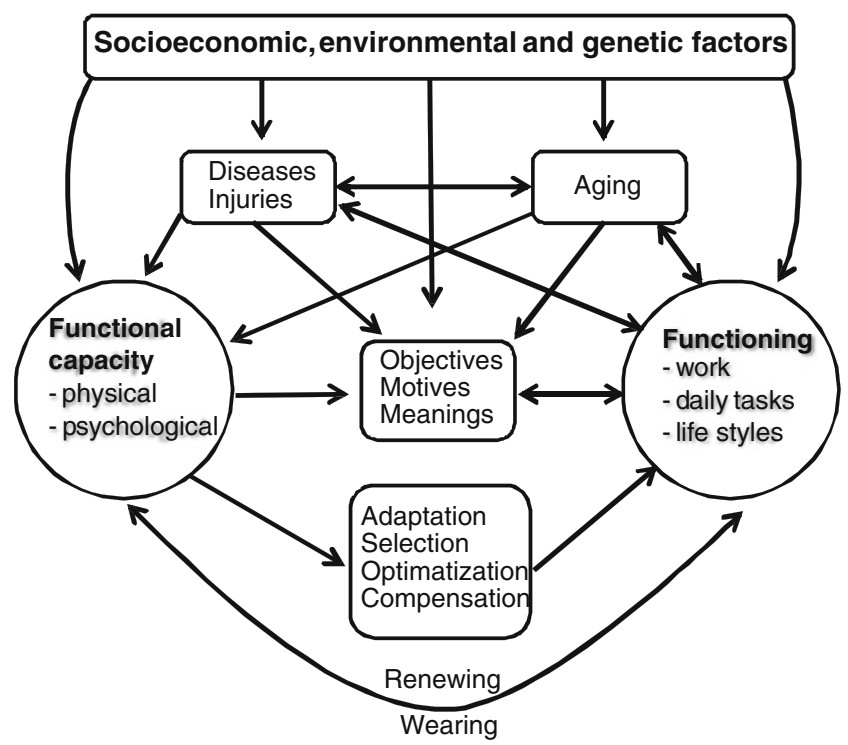

Fig. 2 A holistic view of the relationships between different biological, medical, psychological, social, and environmental factors that may operate at the individual level over the life course 
The most complex and demanding challenge for the life course approach lies in taking a holistic view of people, including a wide range of environmental and individual risk factors and in developing means for effective interventions to reduce or modify such risk factors and behaviors during the different phases of life. This challenge is schematized in Fig. 2.

It is hypothesized that the broader socioeconomic, environmental, and genetic effects, within which individuals live during their life span, cumulatively influence health, health behaviors, functioning, and certain psychological characteristics and measures and their interactions [31]. Particular emphasis is placed on the association between the level and content of functioning and an individual's functional capacity, and on the objectives and motives, and meanings individuals have for different activities. Furthermore, individuals have different abilities for adaptation in the changing situations, and they differ in the capability to select among different activities, optimize resources, and to use compensatory means so as to be able to carry out the most important activities [34]. When age-related decline in repertoire (health and functioning) threatens the individual's capability to attain important goals, it is possible to improve the disturbed balance between repertoire, personal goals, and environment by e.g., rehabilitating functioning, changing goals, and improving the social and physical environment [35].

During recent decades, "there has been a shift from homogeneity, continuity and universality of developmental processes to heterogeneity, discontinuity and context-specific development" [3]. This creates major challenges for both the design and conduct of studies applying a life course approach. It may be difficult to obtain adequate measures of exposures across the life course, without which it is not possible to test the mechanisms underlying either biological exposures or social interventions [36]. Clearly, a range of research designs and methods need to be applied, including not only birth cohort, historical cohort, and record linkage studies but also randomized controlled trials and case control studies. Moreover, it would be important to promote a shift from multidisciplinary to more interdisciplinary research involving a plurality of disciplines where disciplinary boundaries are often muted, and the joint contributions of the synergy are highlighted $[37,38]$.

Bridging the biological, psychological, and social models of the life course approach is expected to help elucidate new mechanisms of disease causation and develop measures for the maintenance of health in aging.

\section{References}

1. Kuh D, New Dynamics of Ageing [NDA] Preparatory Network (2007) A life course approach to healthy ageing, frailty and capability. J Geront Med Sci 62A:717-721
2. Franco OH, Karnik K, Osborne G, Ordovas JM, Catt M, van der Ouderaa F (2009) Changing course in ageing research: the healthy aging phenotype. Maturitas 63:13-19

3. Ben-Shlomo Y, Kuh D (2002) A life course approach to chronic disease epidemiology: conceptual models, empirical challenges and interdisciplinary perspectives. Int J Epidemiol 33:285-293

4. Darnton-Hill I, Nishida C, James WPT (2004) A life course approach to diet, nutrition and the prevention of chronic diseases. Public Health Nutr 7:101-121

5. Clarke AM, Clarke ABD (1976) The formative years? In: Clarke AM, Clarke ABD (eds) Early experience: myth and evidence. The Free Press, New York

6. Baltes PB, Linderberger U, Staudiger UM (1998) Life-span theory in developmental psychology. In: Damon W, Lerner EM (eds) Handbook of child psychology: theoretical models of human development, vol 1. Wiley, New York, pp 1029-1143

7. Hagestad GO, Neugarten BL (1985) Age and the life course. In: Binstock R, Shanas E (eds) Handbook of aging and the social sciences, 2nd edn. Van Nostrand Reinhold Co, New York, pp 35-61

8. Blane D, Netuveli G, Stone J (2007) The development of life course epidemiology. Epidemiol Public Health 55:31-38

9. Barker D (1994) Mothers, babies and diseases in later life. British Medical Journal, London

10. Blane D (2006) The life course, the social gradient, and health. In: Marmot M, Wilkinson RG (eds) Social determinants of health, 2nd edn. Oxford University Press, Oxford, pp 54-77

11. Wadsworth MEJ (1997) Health inequalities in the life course perspective. Soc Sci Med 44:859-869

12. Schöllgen I, Huxhold O, Tesch-Römer C (2010) Socioeconomic status and health in the second half of life: findings from the German Ageing Survey. Eur J Ageing 27:17-28

13. Nybo H, Petersen HC, Gaist D, Jeune B, Andersen K, McGue M, Vaupel JW, Christensen KJ (2003) Predictors of mortality in 2, 249 nonagenarians - the Danish 1905-cohort survey. Am Geriatr Soc 51:1365-1373

14. Kuh DL, Ben-Shlomo Y (1997) A life course approach to chronic disease epidemiology; tracing the origins of ill-health from early to adult life. Oxford University Press, Oxford

15. Kuh D, Ben-Shlomo Y, Lynch J, Hallqvist J, Power C (2003) A glossary of life course epidemiology. J Epidemiol Community Health 57:778-783

16. Leon DA, Kouplikova I, Lit Shell HO et al (1996) Failure to realise growth potential in utero and adult obesity in relation to blood pressure in 50 year old Swedish men. BMJ 312:401-406

17. Eriksson JG, Forsén T, Tuomilehto J, Osmond G, Barker D (2000) Fetal and childhood growth and hypertension in adult life. Hypertension 36:790-794

18. Pickering TG (2001) Why is hypertension more common in African Americans? J Clin Hypertens 3:50-52

19. Berenson GS, Srinivasan SR, Nicklas TA (1998) Atherosclerosis: a nutritional disease of childhood. Am J Cardiol 82:22T-29T

20. Dietz WH (2001) The obesity epidemic in young children. BMJ 322:313-314

21. Bartley M, Blane D, Montgomery S (1997) Health and the life course: why safety nets matter. BMJ 314:1194-1997

22. Blane D, Bartely M, Smith GD (1997) Disease aetiology and materialistic explanation of socioeconomic mortality differentials. Eur J Popul 7:385-391

23. Aboderin I, Kalache A, Ben-Shlomo Y, Lynch JW, Yajnik CS, Kuh D (2002) Life course perspectives on coronary heart disease, stroke and diabetes. Key issues and implications for policy and research. World Health Organization, Geneva

24. Mann SL, Wadsworth MEJ, Colley JRT (1992) Accumulation of factors influencing respiratory illness in members of a national birth cohort and their offspring. J Epidemiol Community Health 46:286-292 
25. Davey Smith G, Hart C, Blane D, Gillis C, Hawthorne V (1997) Lifetime socioeconomic position and mortality: prospective observational study. BMJ 3:547-552

26. Leon DA, Lit Shell HO, Vagero D, Koupilova I, Mos Shen R, Berglund L et al (1998) Reduced fetal growth rate and increased risk of death from ischaemic heart disease: cohort study of 15,000 Swedish men and women born 1915-1929. BMJ 31:241-245

27. Forsén T, Eriksson J, Tuomilehto J, Reunanen A, Osmond C, Barker D (2000) The fetal and childhood growth of persons who develop type 2 diabetes. Ann Intern Med 133:176-182

28. Kivimäki M, Lawlor DA, Juonala M, Smith GD, Elovainio M, Keltikangas-Järvinen L et al (2005) Lifecourse socioeconomic position, $\mathrm{C}$-reactive protein, and carotid intima-media thickness in young adults: the cardiovascular risk in Young Finns Study. Arterioscler Tromb Vasc Biol 25:2197-2202

29. Davey Smith G, Hart C, Blane D, Hole D (1998) Adverse socioeconomic conditions in childhood and cause specific adult mortality: prospective observational study. BMJ 316:1631-1635

30. Kalandar-Zadeh K, Block G, Horwich T, Fonarow GC (2004) Reverse epidemiology of conventional cardiovascular risk factors in patients with chronic heart failure. J Am Cardiol 43:1439-1444

31. Ahmed N, Mandel R, Fain MJ (2007) Frailty: an emerging geriatric syndrome. Am J Med 120:748-753
32. Strandberg TE, Strandberg AY, Salomaa W, Pitkälä KH, Tilvis RS, Sirola J, Miettinen TA (2009) Explaining the obesity paradox: cardiovascular risk, weight change, and mortality during longterm follow-up in men. Eur Heart J 30:1720-1727

33. Shephard RJ (1997) Impact of aging on major physiological systems and rates during submaximal and maximal exercise. In: Shephard RJ (ed) Aging. Physical activity and health. Human Kinetics, Champaign, pp 57-138

34. Baltes PB (1997) On the incomplete architecture of human ontogeny. Selection, optimization, and compensation as foundation of development theory. Am Psychol 52:366-380

35. Heikkinen E (2006) Disability and physical activity in late-liferesearch models and approaches. Eur Rev Aging Phys Act 3:3-9

36. Singhal A, Cole TJ, Lucas A (2001) Early nutrition and preterm infants and later blood pressure: two cohorts after randomized trials. Lancet 357:413-419

37. Ferraro KF, Chan S (1997) Is gerontology a multidisciplinary or interdisciplinary field of study? Evidence from scholarly affiliations and educational programming. In: Ferraro KF (ed) Gerontology; perspectives and issues. Springer, New York, pp 373-387

38. Ferraro KF (2007) Is gerontology interdisciplinary? J Geront B PsySci and SocSci 62:87 